Ann. Sci. forest., 1977, 34 (2), 111-135.

\title{
Les systèmes racinaires de jeunes plants de Hêtre et de Chêne I. - Modification de leur morphogénèse par décapitation d'exîrémités de racines et conséquences pratiques
}

\author{
A. RIEDACKER et $U$. PODA \\ avec la collaboration technique de J. F. MULLER \\ Station de Sylviculture ef de Production. \\ Centre Nalional de Recherches forestières, I.N.R.A., \\ 54280 Champenoux par Seichamps
}

\section{Résumé}

Le pivot et les racines latérales de ces feuillus ont èté décapités à divers stades de développement : (0) immédiatement après la germination (1) à 1 an (3) à 3 ans.

Au stade (0), le pivot de Hêtre décapité une fois régénère en moyenne 5 racines contre 3 pour celui du Chêne; ces pivots de régénération ont chacun une croissance comparable au pivot unique. La répétition des décapitations du pivot du Chêne augmente le nombre de racines régénérées, mais le diamètre de celles-ci diminue.

Au stade (1), le pivot de Hêtre se régénère mal, surtout s'il a êté coupé près du collet ; celui du Chêne se régénère un peu mieux, mais irrégulièrement. Au stade (3), le pivot de Hêtre coupé à moins de 20 centimètres du collet ne se régénère plus, tandis que celui du Chêne régénère essentiellement des racines obliques ou horizontales. Les racines latérales aussi bien au stade (1) que (3) se régénèrent en revanche très bien, sauf si elles ont été sectionnées au ras du pivot; une racine aux caractéristiques analogues remplace la partie décapitée.

Au stade (0), la manipulation des racines ne se répercute ni sur la morfalité, ni sur le taux de survie Aux stades (1) et (3), l'ablalion des racines latérales abaisse en revanche beaucoup plus nettement le taux de survie et le développement aérien que le raccourcissement du pivot.

Pour concentrer près du collet le chevelu racinaire de ces feuillus les auteurs proposent soit de décapiter avant semis les radicules de faines ou glands prégermés, soit d'élever de très jeunes plants en petits paperpots dont on sectionne la base avant repiquage.

Mots clefs : Jeunes plants ; Fagus sylvatica L. ; Quercus pedunculata Ehrh ; Régénération ; Pivots ; Racines latérales ; Relation Racine/Tige ; Débourrement ; Mortalité ; Croissance aérienne ; Méthode d'élevage en pépinière.

\section{Introduction}

Cette étude a été entreprise pour déterminer l'influence de la morphologle juvénile du système racinaire du Hêtre (Fagus sylvatica L.) et du Chêne (Quercus pedunculata Ehrh) sur :

- leur reprise,

- leur croissance immédiatement après transplantation,

- leur croissance et leur stabilité au vent jusqu'au moment de leur exploitation 

fourches,

- leur morphogénèse aérienne et notamment leur tendance à produire des

- les caractéristiques du bois qu'ils produiront.

On peut en effet supposer que selon que le système racinaire explorera simplement les horizons superficiels (cas d'un système racinaire traçant) ou essentiellement les horizons profonds (cas d'un système racinaire ayant seulement des racines pivotantes), ou encore les 2 horizons à la fois, l'intensité et le rythme de la croissance, ainsi que les caractéristiques du bois qui sera produit, risquent de différer.

II convenait donc au préalable de préciser comment on peut pour ces essences obtenir différentes morphologies de système racinaire. L'objct de cette note est de présenter succinclement une partie des résultats de ces études. Certains aspects plus fondamentaux ont déjà été publiés (Riedacker, 1976), ou le seront ultérieurement.

Pour modifier la morphogénèse de ces systèmes racinaires, on peut imaginer :

1) de faire varier les paramètres physiques ou chimiques du milieu extérieur,

2) de pratiquer des ablations des extrêmités du pivot ou des racines latérales, afin de modifier les corrélations au sein du système racinaire ou en vue de provoquer des régénérations de racines.

Nous envisagerons ici surtout l'effet de la seconde pratique.

\section{I. - Influence du substrat (fig. 1)}

Les radicules de glands et de faînos donnent généralement naissance dans les conditions naturelles à un fort pivot portant des racines latérales plus ou moins courtes. Et que le milieu soit facilement pénétrable ou non, tant que le méristème terminal du pivot n'est pas endommagé, les racines latérales de ces 2 essences restent fines, horizontales ou obliques et n'ont qu'un allongement limité ; sur tourbe, perlite et même des schistes feuilletés, les « squelettes» des systèmes racinaires de chênes diffèrent peu. Si le pivot de Chêne rencontre une couche d'argile, il la pénètre ; s'il rencontre un obstacle impénétrable (une pierre, le fond d'une cuvette en matière plastique, etc...) il est dévié, mais sa morphogenèse est peu modifiée. Le pivot de Hêtre réagit sensiblement de la même manière.

Le développement des fines radicelles (sur les racines latérales et le pivot) est en revanche fortement influencé par le milicu de culture. Sur tourbe, ces dernières se développent beaucoup moins que sur sable fin et même que sur sol argileux (fig. 1). Les facteurs responsables de ces différences de développement n'ont toutefois pas encore été mis en évidence.

\section{II. - Décapitations des radicules au cours des semaines suivant la germination}

$$
\text { A. - Hêtre }
$$

\section{Expérimentation.}

Nous avons coupé avec des ciseaux la radicule de plantules semées en serre en mai aux stades suivants :

- Plantules témoins $\left(T_{0}\right)$. 

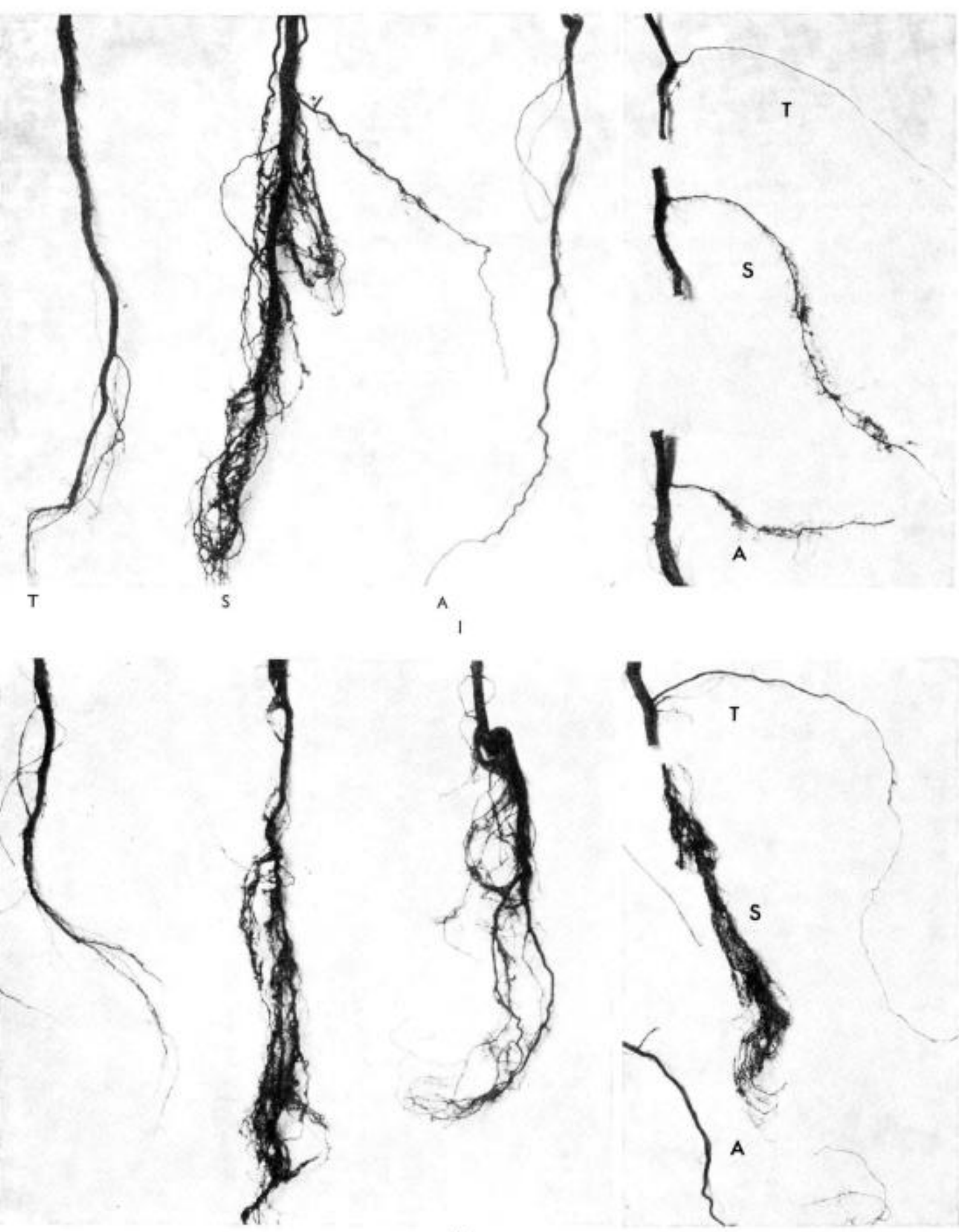

II

FIG. 1. - Systèmes racinaires de plants de Chène (I) el de Hêtre (II) d'un an élevés sur fourbe ( $T$ ), sable fin (S) et sol argileux (A).

Roof systems of one year-old Oak (I) and beech (II) seedlings grown in peat (T), fine sand (S) and clayey soil $(A)$. 
- Plantules ayant 2 cotylédons bien développés et une radicule de 7,5 centimètres de long que l'on coupe à :

- 2,5 centimètres du collet $\left(T_{2,5}\right)$.

- 5 centimètres du collet $\left(T_{5}\right)$,

- 7,5 centimètres du collet $\left(T_{7+5}\right)$.

- Plantules ayant 2 feuilles bien développées at une radicule de 10 à 15 centimètres de long que l'on coupe à :

$-2,5$ centimètres du collet $\left(\mathrm{T}_{2,5}\right)$,

- 7,5 centimètres du collet $\left(T_{7}, 5\right)$.

- Faine venant de germer avec une radicule de 2 à 3 centimètres de long sectionnée à 1 centimètre de la coiffe $\left(\mathrm{T}^{*}\right)$.

Les plants ont été élevés sur tourbe fertilisée (dose 2 proposée par Delran, Garbaye et Le Tacon, 1975), et sur sable pur (à raison de 9 plants par seau et 4 répétitions par traitement) et arrosé avec une solution nutritive préparée à partir de l'eau du robinet par adjonction d'engrais ( $\mathrm{pH}$ 6) selon la méthode Coic et Lesaint (1973).

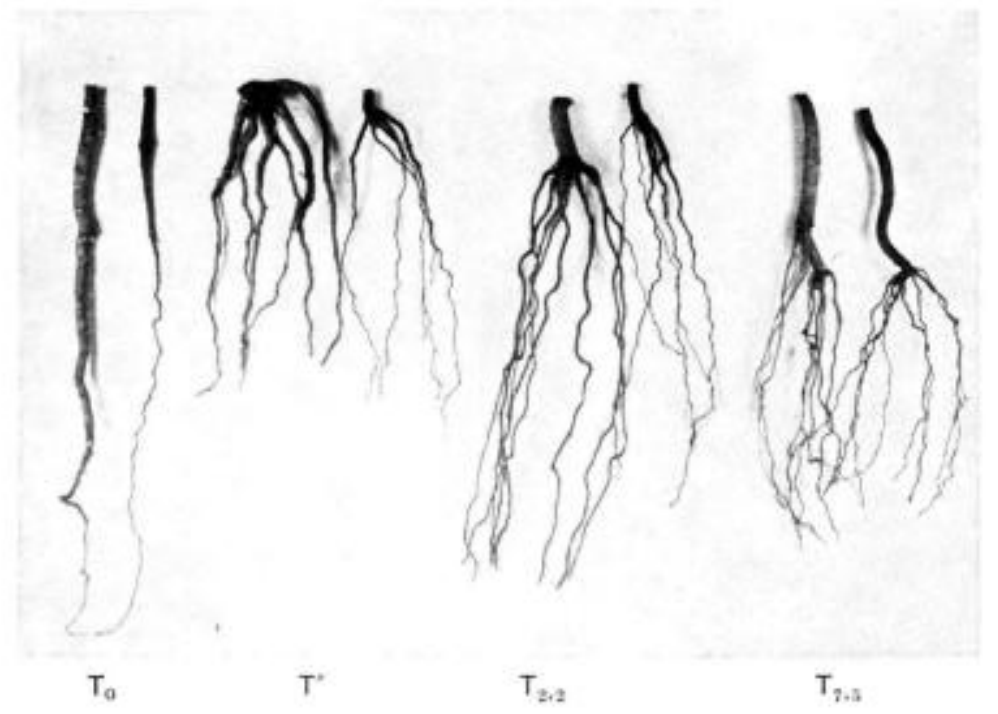

FIG. 2. - Influence de la décapitation de la radicule de Hễtre après la germination sur la morphogénèse de leurs systèmes racinaires (les fines racines ont été enlevées pour permettre une meilleure observation).

$T_{0}$ : Témoin (à gauche dans la tourbe, à droite dans le sable).

$T^{\prime}$ : Avec radicule de $2-3 \mathrm{~cm}$ de long coupée à la germination à $1 \mathrm{~cm}$ de son extrêmité (sur tourbe et sable).

$T_{255}$ : Avec radicule décapitée à $2,5 \mathrm{~cm}$ du collet au stade cotylédonaire (sur tourbe ef sur sable).

$T_{7,5}$ : Comme $T_{2,5}$, mais avec décapitation à $7,5 \mathrm{~cm}$ du collet.

Effect of beech radicle decapitation upon root morphogenesis (fine roots were removed to allow betfer observation).

$T_{0}$ : Control (Left in Peat-Rigth in sand).

$\mathrm{T}^{*}$ : With radicle cut at $1 \mathrm{~cm}$ from the cap when $2-3 \mathrm{~cm}$ long (Left in peat-Right in sand).

$T_{2.5}$ : With radicle cut at $2,5 \mathrm{~cm}$ from the collar at the $\alpha$ cotyledons $\mathrm{x}$ stage.

$T_{7.5}$ : As $T_{2.5}$ but cut at $7,5 \mathrm{~cm}$ from the collar. 
2. Résultats (fig. 2 et tabl. 1).

Après une saison de végétation, il est possible de tirer de l'essai les conclusions suivantes :

- quel que soit le niveau de section du pivot, celui-ci régénère * 2 à 8 racines verticales (moyenne 5) :

- l'allongement et la croissance radiale des racines régénérées sont d'autant plus comparables à ceux du pivot des plants témoins que la section a eu lieu le plus près du collet ;

- la décapitation du pivot effectuée au stade " 2 feuilles " ou au stade « 2 cotylédons » permet d'obtenir le même développement racinaire ;

- le pivot se régénère de la même manière sur sol sableux et sur tourbe : les racines.

- le développement aérien n'est pas influencé par les traitements qu'ont subi

\section{TABLEAU 1}

Influence de divers traitements racinaires

quelques jours après la germination sur le développement des Hêtres

Influence of various treatments on roots of Beech seedlings immediately after germination

\begin{tabular}{|c|c|c|c|c|c|c|c|}
\hline \multirow{2}{*}{\multicolumn{2}{|c|}{$\begin{array}{l}\text { Mesures après une saison de végétation } \\
\text { (Measurements after one growing season) }\end{array}$}} & \multicolumn{6}{|c|}{ Traitement (Treatment) } \\
\hline & & $T_{0}$ & $T_{2,5}$ & $T_{7+5}$ & $T_{2,5}$ & $T_{7.5}$ & $T^{\prime \prime}$ \\
\hline \multirow{3}{*}{$\begin{array}{l}\text { Hauteur en } \mathrm{cm} \\
\text { (Height in } \mathrm{cm})\end{array}$} & Tourbe (Peat)... & 26,8 & 21,3 & 22,5 & 20,9 & 23,6 & - \\
\hline & Sable (Sand)..... & 10.5 & 11,2 & 11,7 & 10,3 & 10,3 & - \\
\hline & $\begin{array}{l}\text { Sol argileux } \\
\text { (Clayey soil)..... }\end{array}$ & 14,1 & - & - & - & - & - \\
\hline \multirow{3}{*}{$\begin{array}{l}\text { Diamètre au collet en mm } \\
\text { sur (Collar diameter in } \\
\mathrm{mm} \text { ) }\end{array}$} & Tourbe (Peat).... & 5 & 4,9 & 4,9 & 4,6 & 4,7 & - \\
\hline & Sable (Sand)..... & 2,5 & 3,6 & 3,3 & 2,9 & 4,3 & - \\
\hline & $\begin{array}{l}\text { Sol argileux } \\
\text { (Clayey soil)..... }\end{array}$ & $\begin{array}{l}3,1 \\
3,1\end{array}$ & $\overline{-}$ & $\overline{-}$ & $\overline{-}$ & $\overline{-}$ & - \\
\hline \multirow{2}{*}{$\begin{array}{l}\text { Nombre moyen de pivots } \\
\text { de remplacement sur } \\
\text { (Mean number of regene- } \\
\text { rated taproots).......... }\end{array}$} & Tourbe (Peat).... & 1 & $\begin{array}{c}6,7 \\
(1,8)\end{array}$ & $\begin{array}{r}5,2 \\
(1,6)\end{array}$ & $\begin{array}{l}6,5 \\
(2,5)\end{array}$ & $\begin{array}{l}5,8 \\
(2,5)\end{array}$ & $\begin{array}{l}5 \\
(1,5)\end{array}$ \\
\hline & Sable (Sand)...... & 1 & $\begin{array}{l}6 \\
(1,9)\end{array}$ & $\begin{array}{c}3,9 \\
(1,3)\end{array}$ & $\begin{array}{l}5.7 \\
(2)\end{array}$ & $\begin{array}{l}3,6 \\
(2)\end{array}$ & $\begin{array}{c}4,5 \\
(1,2)\end{array}$ \\
\hline
\end{tabular}

( ) Ecart-type (Standerd error) - Non mesuré ou traitement manquant (Not measured or tested) (Les différences entre colonnes ne sont pas significatives au seuil de 5 p. 100) (Differences between columns are not significant at the 5 p. 100 level.)

Cet essai a été partiellement reproduit à une plus grande échelle en 1975 en plein air et sur tourbe fertilisée (tabl. 2) ; les résultats obtenus confirment ceux qui ont été énoncés ci-dessus.

* Le terme régénération a été utilisé dans le sens $\alpha$ apparition de nouvelles racines », ce qui ne signifie pas nécessairement qu't y a initiation de nouvelles racines. 


\section{TABLEAU 2}

Caractéristiques moyennes des plants moyens élevés en plein air sur tourbe après un nombre variable de décopitation de la rodicule

Characteristics of mean seedlings grown outdoor in peat after various root treatments

\begin{tabular}{|c|c|c|c|c|c|c|c|}
\hline Essence (Species) & \multicolumn{3}{|c|}{ Hêtre (Beech) } & \multicolumn{3}{|c|}{ Chêne pédonculé (Oak) } & \multirow{3}{*}{ Différences } \\
\hline \multirow[b]{2}{*}{$\begin{array}{l}\text { (Caracteristics) } \\
\text { Caractéristiques }\end{array}$} & \multirow{2}{*}{$\begin{array}{l}\text { Témoin } \\
\text { (Control) }\end{array}$} & \multirow{2}{*}{$\begin{array}{l}\text { Radicule } \\
\text { coupée } \\
\text { une fois } \\
\text { (Tap root } \\
\text { cut once) }\end{array}$} & \multirow[t]{2}{*}{ Différences } & \multirow{2}{*}{$\begin{array}{l}\text { Témoin } \\
T_{0} \\
\text { (Control) }\end{array}$} & \multicolumn{2}{|c|}{$\begin{array}{l}\text { Radicule } \\
\text { recoupée } \\
\text { Radicle cut }\end{array}$} & \\
\hline & & & & & $\frac{1}{T_{1}}$ & $\mathrm{~T}_{2}^{2}$ & \\
\hline 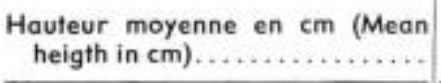 & 18,9 & 17.8 & NS & 29,4 & 33,8 & 29,8 & NS \\
\hline 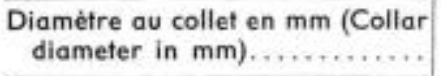 & 4,8 & 4,8 & NS & 7,9 & 7,7 & 7.7 & NS \\
\hline $\begin{array}{l}\text { Poids sec des racines entre } 0 \text { et } \\
20 \mathrm{~cm} \text { du collet en } \mathrm{g} \text { (Dry weigth } \\
\text { of root portion befween } 0 \text { and } \\
20 \mathrm{~cm} \text { from the collar in g)..... }\end{array}$ & 1,03 & 0.75 & NS & 5,77 & 8,12 & 4,44 & $\begin{array}{l}T_{1}>T_{0} \\
\text { ef } T_{2}\end{array}$ \\
\hline $\begin{array}{c}\text { Nombre moyen de pivots (Mean } \\
\text { number of taproots.......... }\end{array}$ & 1 & $\begin{array}{c}5 \\
\text { (entre } 3 \\
\text { à } 8)\end{array}$ & - & 1 & $\left(2 \stackrel{3}{a}{ }^{5}\right)$ & $(3 \stackrel{5}{a} 7)$ & s \\
\hline 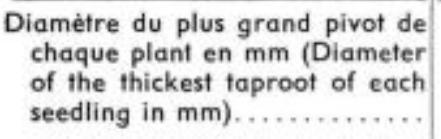 & - & - & & 3 & - & 1,3 & S \\
\hline
\end{tabular}

NS - non significatif (non significant) (seuil de 95 p. 100).

$\mathrm{S}=$ significatif (significant) (seuil de 95 p. 100).

\section{B. - Chêne pédonculé}

La simple décapitation du pivot de plantules de Chêne ne permet d'obtenir en moyenne qu'1 à 2 pivots de remplacement si celle-ci a lieu à 1 centimètre de sa coiffe, et 3 (2 à 5) si elle est pratiquée dans une zone oủ existent des racines latérales (Champagnat et coll., 1974). Cette modification ne nous ayant pas paru suffisante, nous avons redécapité une ou plusieurs fois les pivots de remplacement qui naissent au fur ef d̀ mesure.

\section{Expérimentation.}

En juin 1975, nous avons semé en serre, sur tourbe fertilisée, des glands ayant subi les traitements suivants :

- Glands avec radicule non décapitée (traitement $T_{0}$ ).

- Glands avec radicule décapité à 5 centimètres du collet lorsqu'elle mesure entre 7 et 10 centimètres de long (traitement $T_{1}$ ). 
- Plantules dont le gland a subi le traitement $T_{1}$ et dont les pivots de remplacement ont été redécapités à 1 centimètre de la section précédente, lorsque leur longueur atteignait 5 à 6 centimètres (traitement $T_{2}$ ).

- Plantules ayant subi le traitement $T_{2}$ et dont on a décapité les pivots de remplacement une nouvelle fois (traitement $T_{3}$ ).

- Plantules ayant subi le traitement $T_{3} \in t$ dont on a décapité les pivots de remplacement une nouvelle fois (traitement $T_{1}$ ).

Chaque traitement comprenant 4 seaux de 9 plants était arrosé régulièrement par une solution nutritive Coïc.

Outre ces essais en serre, les traitements 0,1 et 2 ont été répétés la même année, en plein air, sur tourbe fcrtilisée ( 150 plants par traitement).

\section{Résultats.}

De ces essais (fig. 3 et tabl. 2 ), se dégagent les résultats suivants :

- Une décapitation unique du pivot permet d'obtenir une régénération moyenne de 3 pivots, dont le développement de chacun est comparable au pivot qu'ils remplacent; le poids moyen des racines par plant entre 0 et 20 centimètres du collet augmente alors significativement ( 8,1 grammes contre 5,8 grammes). Ces pivots de remplacement ont une direction de croissance proche de la verticale.

- Lorsqu'on répète la décapitation, les racines régénérées deviennent plus fines (diamètre moyen du plus gros pivot 1,3 millimètre contre 3 millimètres chez les témoins) ; le poids moyen des racines entre 0 et 20 centimètres du collet redevient analogue aux témoins, le nombre de pivots de remplacement continue par contre

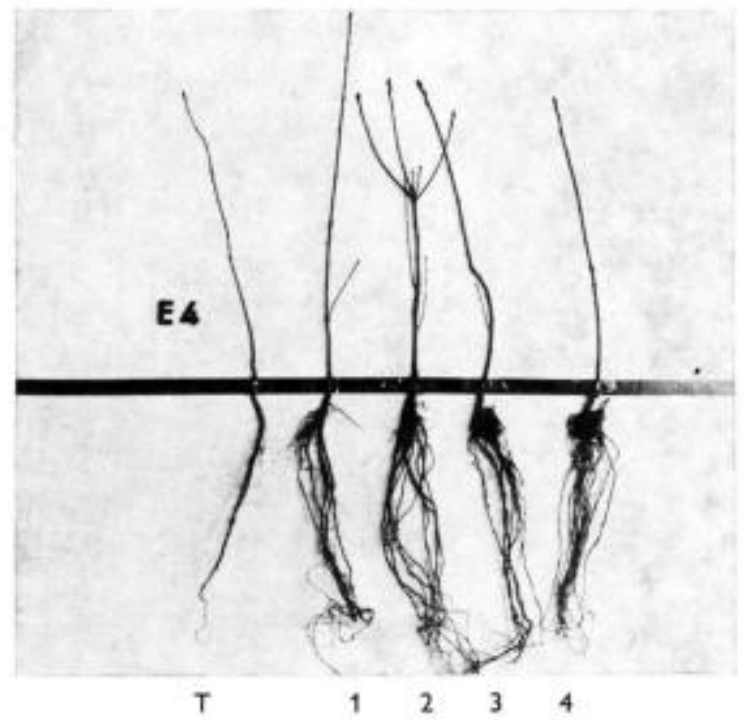

FIG. 3. - Systèmes racinaires de Chênes dont le pirot a été recoupé (1), (2) (3) ou (4) fois au cours des semaines ayant suivi la germination et témoin $(T)$.

Oak root systems with the toproots cut (1), (2), (3) or (4) times during the weeks following germination and control $(T)$. 
à s'élever ; pour 2 décapitations, on obtient en moyenne 5 pivots de remplacement (3 à 7 ) contre 3 avec une seule décapitation. Après 3 ef 4 décapitations, on obtient respectivement entre 4 à 8 racines et 8 à 11 racines remplaçant le pivot primitif. Comme chez le Hêtre, le traitement que subissent les racines n'influe pas significativement sur le développement aérien.

\begin{tabular}{|c|c|c|c|c|c|}
\hline Traitement & $T_{0}$ & $\mathrm{~T}_{1}$ & $T_{2}$ & $\mathrm{~T}_{3}$ & $T_{4}$ \\
\hline $\begin{array}{l}\text { Hauteur moyenne des plants } \\
\text { en cm après } 1 \text { an.......... }\end{array}$ & 36,2 & 34,8 & 32,6 & 28,7 & 31,6 \\
\hline
\end{tabular}

\section{III. - Décapitation des racines latérales et du pivot de plants d'un an}

\section{A. - Expérimentation}

Des plants de Chêne pédonculé (hauteur moyenne 18 centimètres) et de Hêtre âgés d'un an (hauteur moyenne 17 centimètres), après avoir été arrachés avec beaucoup de précautions, ont subi les traitements indiqués ci-dessous avant d'être replantés fin mars en plein air, à raison de 15 plants par conteneur de plastique contenant un mélange de sable (2/3) et de tourbe (1/3) (4 bacs de $60 \times 40 \times 40$ centimètres par traitement).

Chaque modalité expérimentale est caractérisée ici, ainsi que dans la suite du texte, par le traitement que subissent respectivement le pivot et les racines latérales.

- $T$ et $t$ signifient que respectivement le pivot ef les racines latérales n'ont pas été resectionnés après extraction :

- les chiffres indiquent la longueur du pivot ou des racines latérales en centimètre après le traitement.

Traitements appliqués au système racinaire de plonfs d'un an

Root treatments on one year - old seedlings

\begin{tabular}{|c|c|c|c|}
\hline \multirow{2}{*}{ Traitement (Treatment) } & \multicolumn{3}{|c|}{$\begin{array}{l}\text { Longueur du pivot } \\
\text { (Tap root length) }\end{array}$} \\
\hline & $\mathrm{T}\left({ }^{1}\right)$ & $10 \mathrm{~cm}$ & $5 \mathrm{~cm}$ \\
\hline 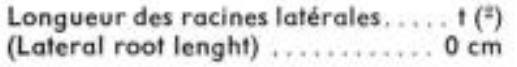 & $\begin{array}{l}T-1 \\
T-0\end{array}$ & $\begin{array}{l}10-t \\
10-0\end{array}$ & $\begin{array}{l}5-1 \\
5-0\end{array}$ \\
\hline $\begin{array}{l}\text { (1) \& Control is for the tap root. } \\
\text { (2) \& Control is for the lateral root. }\end{array}$ & & & \\
\hline
\end{tabular}


En outre, nous avons observé en minirhizotrons (Riedacker, 1974) les modalités de régénération de racines de plants de Chêne et de Hêtre d'un an dont la radicule avait été soit décapitée, soit laissée intacte au moment de la germination (4 plants par traitement et par essence).

Ces plants ont été arrosés par aspersion chaque fois que cela paraissait nécessaire ( 2 à 4 fois par semaine en été).

\section{B. - Résultats}

1. Taux de survie.

Le taux de survie des plants repiqués est peu modifié par le traitement que subissent les racines. II ne diminue que lorsque le pivot, privé de racines latérales, n'a plus qu'une longueur de 5 centimètres (5-0). Dans les autres cas, il est supérieur à 90 p. 100 pour le Hêtre, et compris entre 83 et 98 p. 100 pour le Chêne.

\section{TABLEAU 3}

Martalité des Hêtres et Chênes d'un an ayant subi divers traitements racinaires

Beech and Oak one year old seedling morlality

\begin{tabular}{|c|c|c|c|c|c|}
\hline \multirow{2}{*}{$\begin{array}{l}\text { Traitements } \\
\text { (Treatment) }\end{array}$} & \multicolumn{2}{|c|}{$\begin{array}{l}\text { Mortalité } \\
\text { en p. } 100 \\
\text { (Mortality) }\end{array}$} & \multirow{2}{*}{$\begin{array}{l}\text { Traitements } \\
\text { (Treatment) }\end{array}$} & \multicolumn{2}{|c|}{$\begin{array}{l}\text { Mortalité } \\
\text { en p. } 100 \\
\text { (Mortality) }\end{array}$} \\
\hline & $\begin{array}{l}\text { Chêne } \\
\text { (Oak) }\end{array}$ & $\begin{array}{l}\text { Hêtre } \\
\text { (Beech) }\end{array}$ & & $\begin{array}{l}\text { Chêne } \\
\text { (Oak) }\end{array}$ & $\begin{array}{c}\text { Hêtre } \\
\text { (Beech) }\end{array}$ \\
\hline$T-1 \ldots \ldots \ldots$ & 2 & 3 & T-0, & 10 & 3 \\
\hline $10-t \ldots \ldots \ldots$ & 10 & 0 & $10-0 \ldots \ldots \ldots$ & 17 & 7 \\
\hline $5-1 \ldots \ldots \ldots$ & 15 & 8 & $5-0 \ldots \ldots \ldots$ & $38 *$ & $22=$ \\
\hline
\end{tabular}

- Seuls résultałs significativement différents des autres (Only these figures differ significantiy from the others.)

\section{Développement aérien.}

La mutilation des racines se répercute en revanche fortement sur le développement foliaire (tabl. 4). Dans les conditions de l'expérience, ces différences se maintiennent durant 3 ans ; l'ablation complète des racines latérales est donc une opération préjudiciable dans tous les cas (traitement T-0, 10-0 et 5-0).

\section{Morphogenèse du système racinaire.}

Les observations des racines après une saison de végétation, ainsi que l'observation de la régénération des racines en minirhizotrons, permettent de constater que :

- Les pivots de Hêtres témoins coupés à 10 centimètres ou 5 centimètres du collet se cicatrisent souvent mal, forment un cal et quelquefois 2 ou 3 racines obliques en arrière du cal ; la section de la radicule de plantules âgées de seulement quelques 


\section{TABLEAU 4}

Poids sec moyen de feuilies par plant en $g$

Mean leaf dry weight per seedling in $g$ Hëtre (Beech)

\begin{tabular}{|c|c|c|c|}
\hline $\begin{array}{l}\text { Traitement } \\
\text { (Treatment) }\end{array}$ & $\begin{array}{l}\text { Poids après } 2 \text { ans en } g^{*} \\
\text { (Weight after } 2 \text { years in } g^{*} \text { ) }\end{array}$ & $\begin{array}{l}\text { Traitement } \\
\text { (Treatment) }\end{array}$ & $\begin{array}{l}\text { Poids après } 2 \text { ans en } \mathrm{g} \\
\text { (Weight after } 2 \text { years in } \mathrm{g} \text { ) }\end{array}$ \\
\hline T-t $\ldots \ldots \ldots \ldots$ & 0.97 & $T-0 \ldots \ldots \ldots$ & 0,41 \\
\hline $10-t \ldots \ldots \ldots \ldots$ & 0,47 & $10-0 \ldots \ldots$ & 0,28 \\
\hline 5-t ............... & 0,23 & $5-0, \ldots \ldots$ & 0,19 \\
\hline
\end{tabular}

* Les feuilles ont été récoltées trop tardivement à la fin de la première année. Leaves were collected too late the first year after transplantation.

Chêne (Oak)

\begin{tabular}{|c|c|c|c|c|c|}
\hline \multirow{2}{*}{$\begin{array}{l}\text { Traitement } \\
\text { (Treatment) }\end{array}$} & \multicolumn{2}{|c|}{$\begin{array}{l}\text { Poids après } \\
\text { (Weight after) }\end{array}$} & \multirow{2}{*}{$\begin{array}{l}\text { Traitement } \\
\text { (Treatment) }\end{array}$} & \multicolumn{2}{|c|}{$\begin{array}{l}\text { Poids après } \\
\text { (Weight after) }\end{array}$} \\
\hline & $\begin{array}{c}1 \text { an } \\
\text { (1 year) }\end{array}$ & $\begin{array}{c}3 \text { ans } \\
\text { (3 years) }\end{array}$ & & $\begin{array}{c}1 \text { an } \\
(1 \text { year })\end{array}$ & $\begin{array}{c}3 \text { ans } \\
\text { (3 years) }\end{array}$ \\
\hline$T-t \ldots \ldots \ldots$ & 0,92 & 3,93 & $T-0 \ldots \ldots$ & 0.74 & 2,75 \\
\hline $10-1 \ldots \ldots \ldots$ & 0,79 & 3,79 & $10-0 \ldots \ldots \ldots$ & 0,38 & 1,71 \\
\hline $5-1 \ldots \ldots \ldots$ & 0,54 & 3,14 & $5-0 \ldots \ldots$ & 0,74 & 1.80 \\
\hline
\end{tabular}

semaines donnait naissance à beaucoup plus de racines verticales. Des plants traités selon cette dernière méthode, c'est-à-dire ayant plusieurs pivots, régénèrent plus fréquemment des racines.

- Chez le Chêne, les modifications dues à l'âge sont moins importantes; les pivots de plants d'un an sectionnés à 20 centimètres du collet, tout comme les radicules émergeant du gland, régénèrent 2 à 3 racines légèrement obliques, près de la section (fig. 4).

Lorsque la radicule a été décapitée une seule fois au moment du semis, les pirots de remplacement se comportent sensiblement comme le pivot d'un plant à pivot unique; mais le nombre fotal de racines régénérées par plants est alors beaucoup plus grand (6 contre 2 ou 3 ).

Les plants, dont la radicule a été recoupée 2 fois ou davantage ne régénèrent, lorsque leurs racines sont coupées à 20 centimètres du collet à l'âge d'un an, qu'une seule racine (fig. 4). Elles se comportent alors de ce point de vue comme les racines latérales ne régénérant qu'une seule racine lorsqu'elles ont été décapitées. Les racines latérales, tant du Chêne que du Hêtre se régénèrent très bien, à condition qu'elles n'aient pas été sectionnées au ras du pivot. 


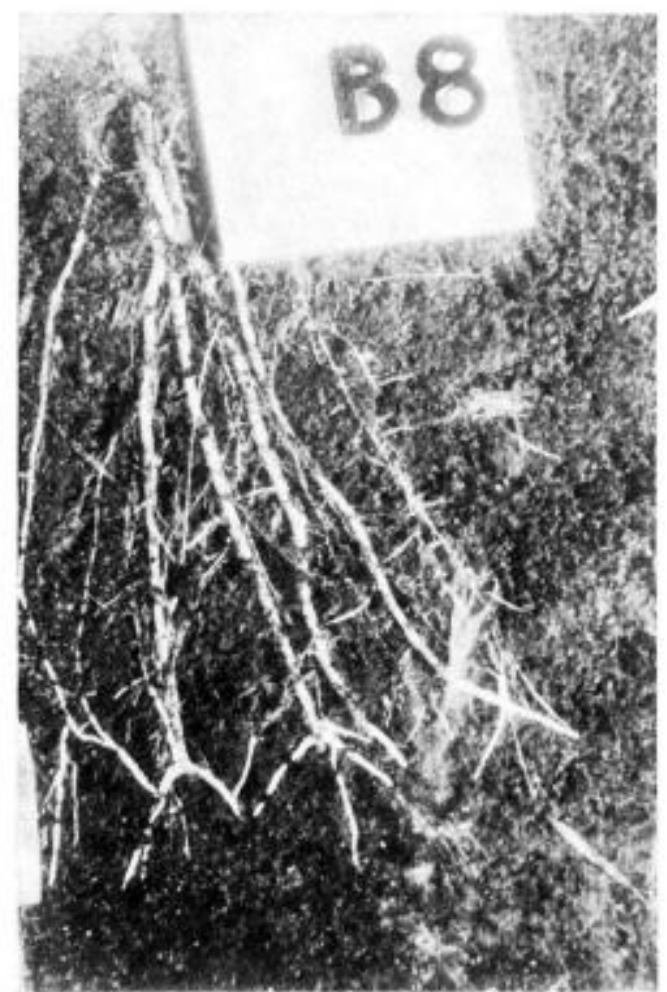

(1)

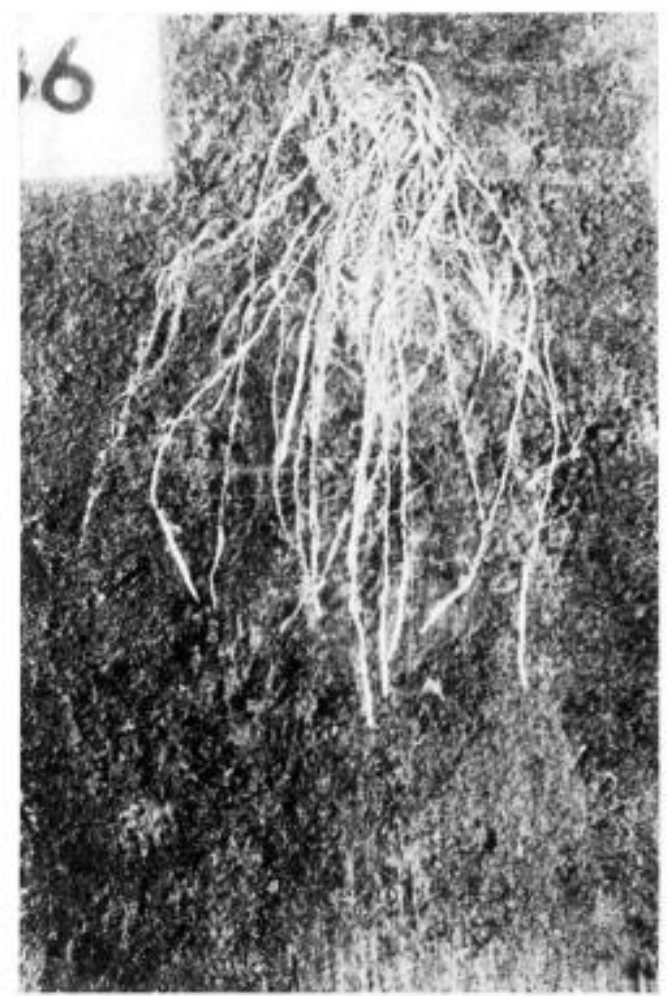

(2)

FIG. 4. - Règénération en minirhizotrons de racines de Chêne d'un an sectionnées à $20 \mathrm{~cm}$ du collet.

(1) Plant dont le pivot a étè coupé une fois immédiatement après la germination ; la croissance radiale ef la régénération de chaque pivot sont comparables à celle d'un pivot d'un plant à un seul pivot.

(2) Plant dont le pivot a été coupé deux fois immédiatement après la germination ; remarquer le diamètre plus fin des « pivots $x$ et ne régénérant en général qu'une racine contre deux à trois précédemment.

Regeneration of roofs offer decapitation at $20 \mathrm{~cm}$ from the collar of one year old oak seedlings in minirhizotrons.

(1) Seedling with a fap root cut once soon affer germination; radial growth of each faproot and regeneration are similar to that of a tap root of plants with a single taproot.

(2) Seedling with a taproot cut twice after germination; $\approx$ taproots $n$ are more numerous but thinner and each of them regenerates only one root, instead of two or three as in the former case.

\section{IV. - Décapitation des racines latérales et du pivot de plants de 3 ans}

\section{A. - Expérimentation}

Comme précédemment, nous avons plus ou moins mutilé les systèmes racinaires de Hêtre et de Chêne ( $40 / 60$ ans) âgés de 3 ans. Les divers traitements figurent dans le tableau-ci-dessous. 


\begin{tabular}{|c|c|c|c|c|}
\hline \multirow{2}{*}{$\begin{array}{l}\text { Longueur des racines latérales } \\
\text { (Laferal root length) }\end{array}$} & \multicolumn{4}{|c|}{ Longueur du pivot (Tap roof length) } \\
\hline & $\mathrm{T}$ & $20 \mathrm{~cm}$ & $10 \mathrm{~cm}$ & $5 \mathrm{~cm}$ \\
\hline \multirow{2}{*}{ t, $\ldots \ldots \ldots \ldots \ldots \ldots \ldots \ldots \ldots}$, & $T-1$ & $20-1$ & $10-t$ & $5-t$ \\
\hline & & $(15-15)$ & $(10-10)$ & $\overline{(5-5)}$ \\
\hline $5 \mathrm{~cm} \ldots \ldots \ldots \ldots$ & T-5 & $20-5$ & $10-5$ & $5-5$ \\
\hline $0 \mathrm{~cm} \ldots \ldots \ldots \ldots \ldots \ldots \ldots \ldots \ldots$ & $T-0$ & $20-0$ & $10-0$ & 5.0 \\
\hline $\begin{array}{l}\left.\text { 1) Yoir note \& III. A ( }{ }^{3}\right) \text {. See s III. A. } \\
\text { Traitements differents du Chène (Spe }\end{array}$ & ea & or oak & ngs). & \\
\hline
\end{tabular}

Ces Hêtres et Chênes dont les traitements racinaires ont été schématisés sur les figures 6 et 8 ont été repiqués sur un milieu identique aux plants d'un an, à raison de 6 plants par poubelle ( 4 poubelles par traitement). Les fréquences d'arrosage ont par ailleurs été identiques à celles des plants d'un an.

La variante appliquée aux racines de Chêne s'explique par la proportion importante de pivots bifides et même trifides que comportait le lot expérimental.

\section{B. - Résultats}

1. Morphogénèse des systèmes racinaires (fig. 5,7 et 9).

Les racines latérales, tant de Hêtre que de Chêne, se régénèrent généralement bien, sauf si elles ont été coupées au ras du pivot.

Le pivot de Hêtre ne se régénère en revanche pas, sauf s'il a été coupé dans sa partie la plus fine à une distance du collet supérieure ou égale à 20 centimètres (fig. 5) ; s'il a été coupé plus près du collet, il n'apparaît le plus souvent qu'un cal cicatriciel sur la section. Selon Dusek (1967), le pivot de Hêtre se régénère rarement lorsque son diamètre au niveau de la section est supérieur à 6-7 millimètres.

Le pivot de Chêne peut se régénérer, quelle que soit la distance de la section au collet (fig. 7) ; la régénération est cependant irrégulière ef lorsqu’elle se produit, les racines qui naissent sont souvent obliques, parfois horizontales, mais rarement verticales.

2. Taux de mortalité.

Contrairement à ce qui avait été observé précédemment, la mutilation plus ou moins importante des racines affecte profondément les chances de survie des plants.

Hêtre (fig. 6).

Ainsi qu'on peut le constater sur la figure 6 , la section des racines latérales à 5 centimètres ou plus du pivot n'affecte pratiquement pas le taux de survie tant qu'elles sont « suffisamment » abondantes ; mais, lorsque le pivot n'a plus que 5 centimètres de long, la présence de longues racines latérales (non coupées à 5 centimètres du pivot) peut néanmoins avoir un effet bénéfique sur la survie (cas du traitement 5-t). L'ablation complète des racines latérales augmente en revanche fortement le taux de mortalité, quel que soit par ailleurs le traitement que subit le pivot. Dans certains cas, ce taux peut atteindre 100 p. 100 après 2 saisons de végétation. 

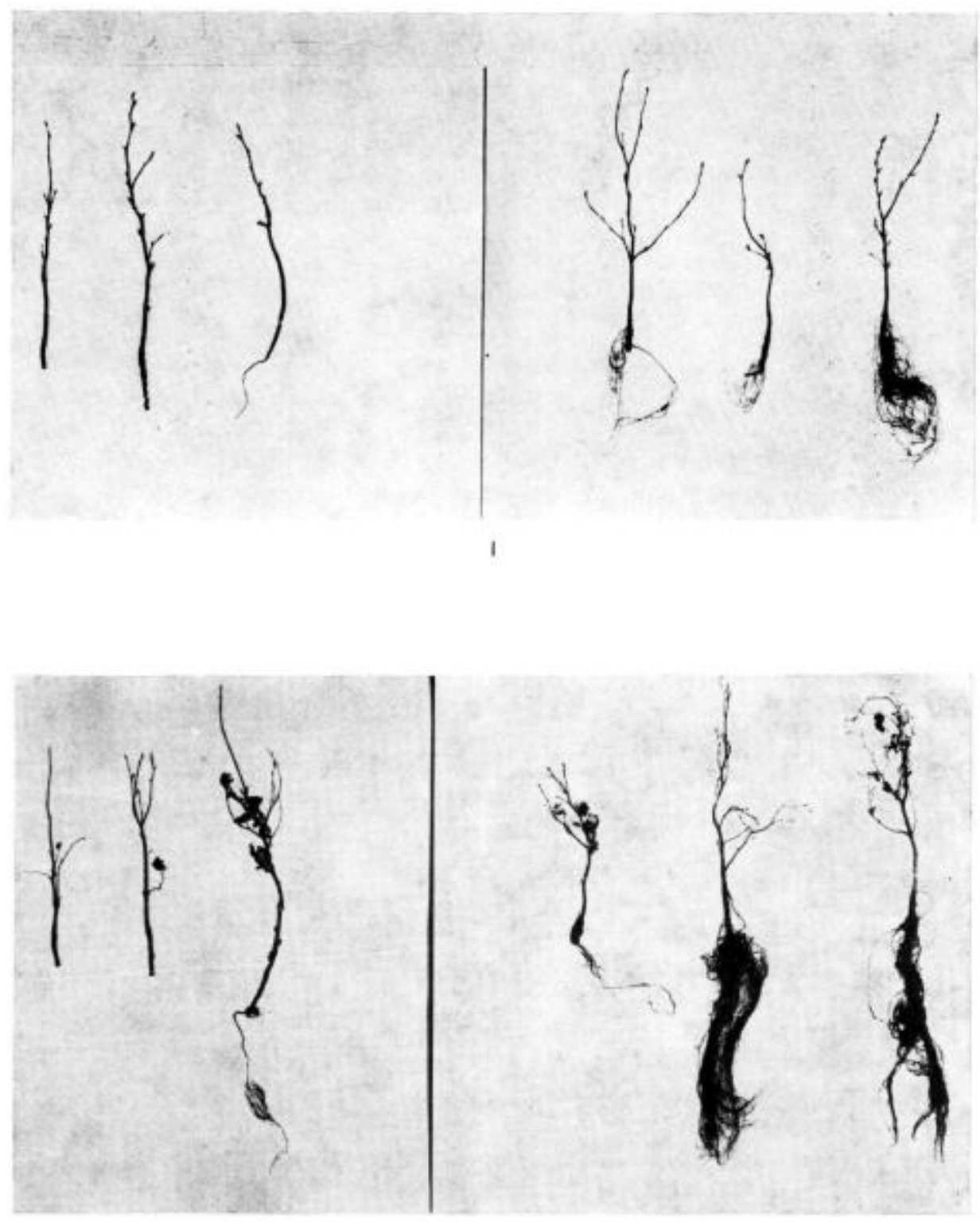

II

FIG. 5. - Régénération des racines de Hêtres de 3 ans ayant subi diverses mutilations (voir début du $\S$ IV et fig. 6).

I. Au moment de la mise en ferre.

II. Après un an.

Root regeneration of 3 years oid beech seedlings with decapited roots (see the beginning of $\S$ IV and fig. 6).
I. Before planting.
II. After one year, 
HETRE
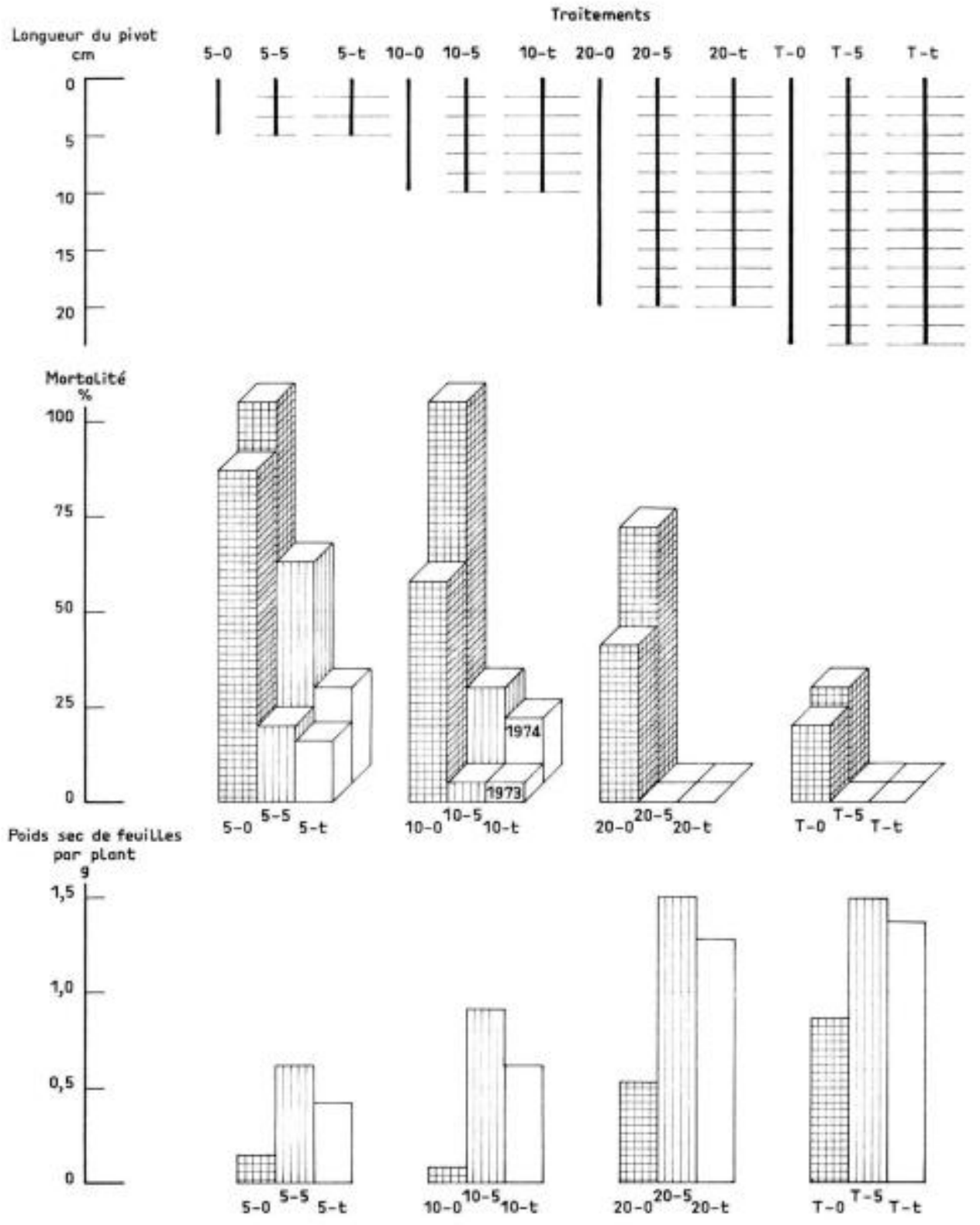

FIG. 6. 

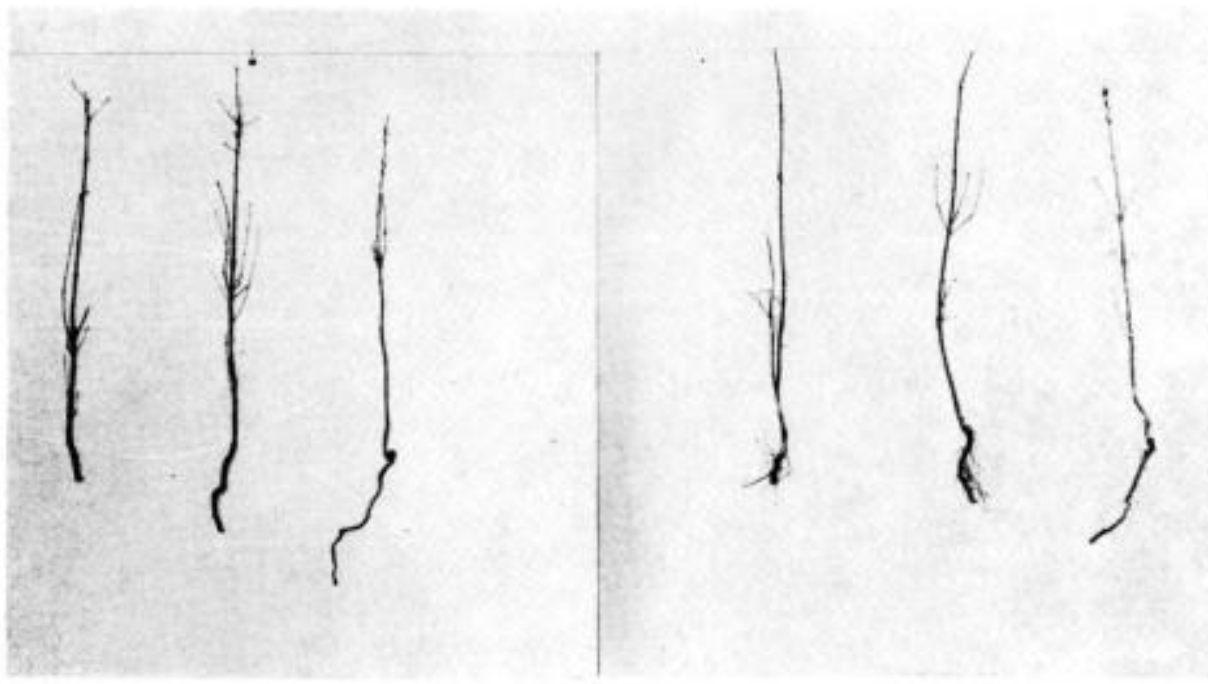

1
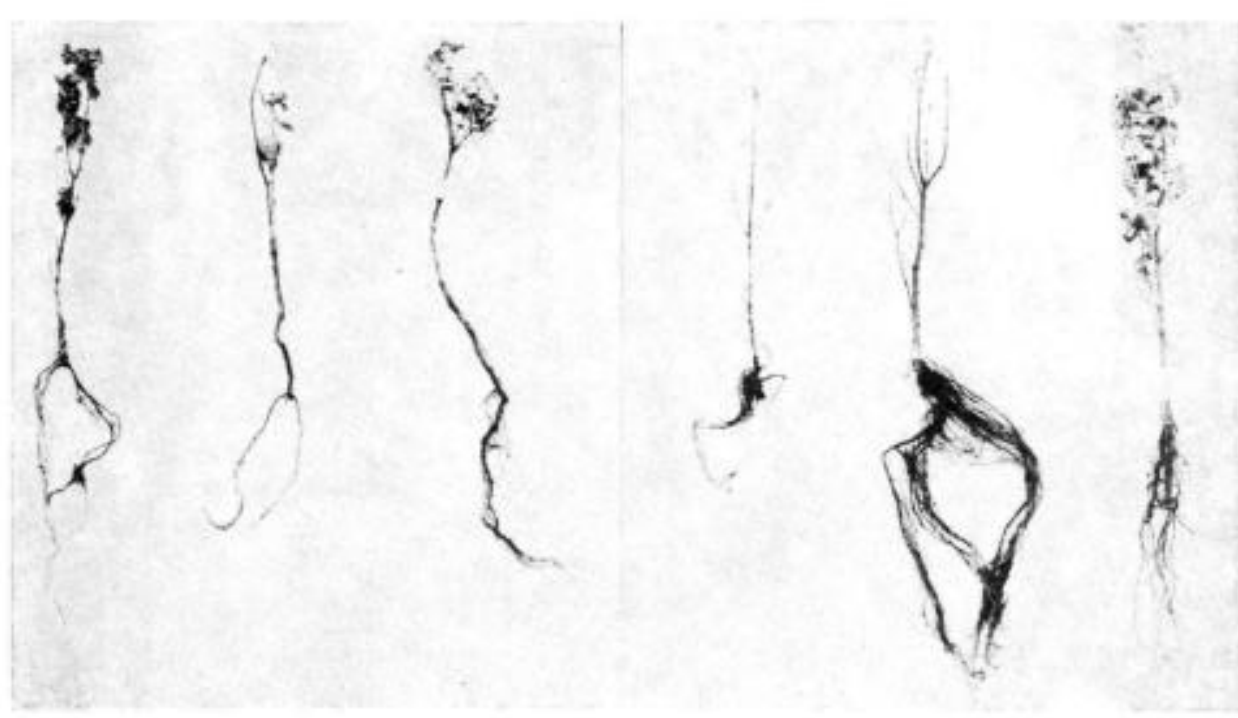

FIG. 7. - Régénérotion de racines de Chênes de 3 ans ayant subi diverses mutilations (voir début du §̧ IV et fig. 8).

I. Au moment de la mise en place.

II. Après un an.

Root regeneration of 3 years old seedlings with decapited roots (see the beginning of $\$$ IV and fig. 8).

I. Before planting.

II. After one year. 
Chêne (fig. 8).

Même lorsque les racines sont peu mutilées, le taux de mortalité est, après un an, généralement plus élevé que celui des Hêtres. La suppression de toutes les racines latérales réduit systématiquement les chances de survie; des plants peuvent d'ailleurs ne mourir qu'au cours de la $2^{2}$ saison de végétation.

\section{Débourrement.}

Au cours des printemps 1973, c'est-à-dire après la mise en place de l'expérience, ef 1974, le débourrement des plants a été observé 2 tois par semaine et nous avons considéré comme débourré tout plant dont au moins un bourgeon avait débourré.

La date moyenne de débourrement désigne la date déterminée graphiquement, à laquelle 50 p. 100 des plants vivants ont débourré.

Le débourrement n'est pas modifié par la décapitation du pivot (colonne 1) ; en revanche, lorsqu'on enlève les racines latérales, celui-ci peut être reterdé jusqu'à 30 jours (colonne 3), surtout chez le Hêtre. Après une saison de végétation, le traitement n'influe plus sur le débourrement.

\section{TABLEAU 5}

Date moyenne de débourrement des plants après mise en place de l'expérience

Mean dafe for budbreak of beech seedlings just after transplantation Hêtre (Beech)

\begin{tabular}{cccccc}
\hline $\begin{array}{c}\text { Traitement } \\
\text { (Treatment) }\end{array}$ & Date & $\begin{array}{c}\text { Traitement } \\
\text { (Treatment) }\end{array}$ & Date & $\begin{array}{c}\text { Traitement } \\
\text { (Treatment) }\end{array}$ & Date \\
\hline T-t & $18-5$ & $T-5$ & $25-5$ & $T-0$ & $11-6$ \\
$20-t$ & $19-5$ & $20-5$ & $30-5$ & $20-0$ & $14-6$ \\
$10-t$ & $19-5$ & $10-5$ & $30-5$ & $10-0$ & $13-6$ \\
$5-t$ & $20-5$ & $5-5$ & $13-6$ & $5-0$ & $25-6$ \\
\hline \hline
\end{tabular}

Chêne (Oak)

\begin{tabular}{cccccr}
\hline $\begin{array}{l}\text { Traifement } \\
\text { (Treatment) }\end{array}$ & Date & $\begin{array}{c}\text { Traitement } \\
\text { (Treatment) }\end{array}$ & Date & $\begin{array}{c}\text { Traitement } \\
\text { (Treatment) }\end{array}$ & Date \\
\hline T-t & $27-5$ & T-5 & $4-6$ & T-0 & $11-6$ \\
$15-15$ & $27-5$ & $15-5$ & $27-5$ & $15-0$ & $30-6$ \\
$10-10$ & $27-5$ & $10-5$ & $29-5$ & $10-0$ & $9-6$ \\
& & $5-5$ & $2-6$ & $5-0$ & $21-6$ \\
\hline \hline
\end{tabular}

\section{Développement aérien.}

a) Production foliaire.

Sur les figures 6 et 8 , nous avons également représenté les masses foliaires produites par les plants moyens ayant subi les divers traitements, après une saison de végétation. 
CHENE
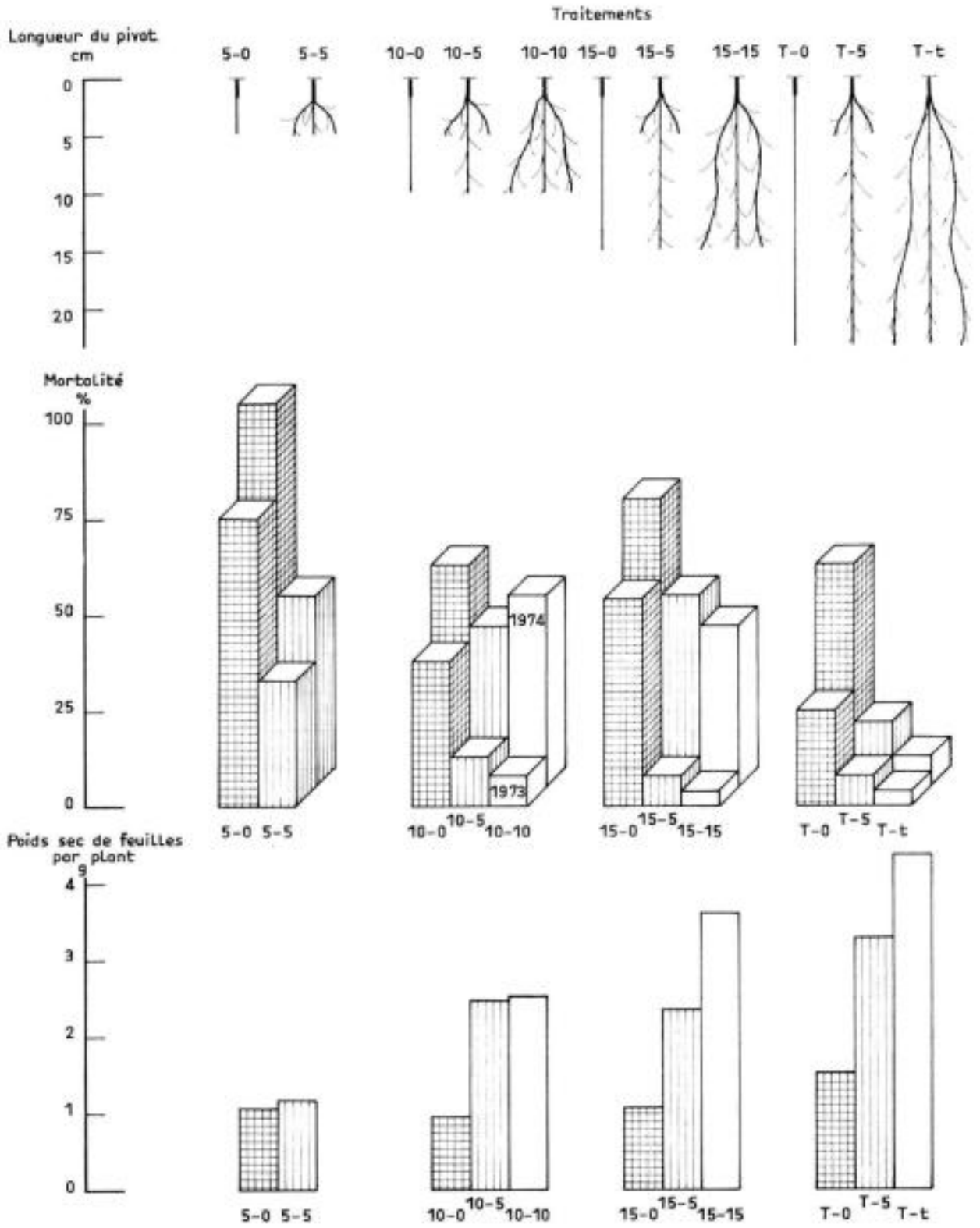

FIG. 8. 
L'ablation totale des racines latérales du Hêtre se traduit dans tous les cas par une forte réduction de la production de feuilles ; très souvent, la cime sèche (fig. 5) et seuls les bourgeons de la base débourrent. Ce phénomène peut toutefois être contrecarré par la suppression des bourgeons de la base et la conservation seulement des 3 ou 4 bourgeons proches du sommet.

Lorsque les racines latérales peuvent se régénérer (section à 5 centimètres ou plus du pivot), le développement foliaire est pour une longueur de pivot donnée voisin de celui des plants ayant des racines latérales plus longues : le « rafraîchissement» des racines latérales semblerait même avoir un effet très légèrement bénéfique. Peut-être cela peut-il s'expliquer par l'extrême difficulté qu'il y a à planter sans déformation des racines latérales longues (dont une partie a pourri dans nos conditions d'élevage).

La mutilation des racines de Chêne entraine dans tous les cas une réduction de la masse de feuilles produites. Le raccourcissement des racines latérales (T-5 : $15-5 ; 10-5 ; 5-5)$ et encore davantage la suppression complète de toutes les racines latérales, c'est-à-dire à la fois les courtes radicelles et les pivots surnuméraires, affectent fortement le développement foliaire.

b) Accroissement au collet (tabl. VI).

Le traitement des racines a un effet sur l'accroissement au collet analogue à celui sur la production foliaire; l'ablation des racines latérales à 5 centimètres du pivot ne diminue sensiblement la croissance au collet que du Chêne. En revanche, l'ablation totale des racines latérales diminue fortement l'accroissement au collet tant du Chêne que du Hêtre.

Là encore l'ablation des racines latérales se traduit par une très forte diminution de la croissance en hauteur.

TABLEAU 6

Accroissement relatif du diamètre au collet au cours de l'année suivant le traitement

Relative collar diameter increment during the year following transplantation Hêtre (Beech)

\begin{tabular}{rrrrrr}
\hline $\begin{array}{c}\text { Traitement } \\
\text { (Treatment) }\end{array}$ & p. 100 & $\begin{array}{r}\text { Traitement } \\
\text { (Treatment) }\end{array}$ & p. 100 & $\begin{array}{r}\text { Traitement } \\
\text { (Treatment) }\end{array}$ & p. 100 \\
\hline T-t & 16 & T-5 & 13 & T-0 & 0.5 \\
$20-t$ & 21 & $20-5$ & 21 & $20-0$ & 4 \\
$10-t$ & 11 & $10-5$ & 10 & $10-0$ & 0 \\
$5-t$ & 4 & $5-5$ & 2 & $5-0$ & 0 \\
\hline
\end{tabular}

Chêne (Oak)

\begin{tabular}{ccrccc}
\hline $\begin{array}{c}\text { Traitement } \\
\text { (Treatment) }\end{array}$ & p. 100 & $\begin{array}{c}\text { Traitement } \\
\text { (Treatment) }\end{array}$ & p. 100 & $\begin{array}{c}\text { Traitement } \\
\text { (Treatment) }\end{array}$ & p. 100 \\
\hline T-t & 22 & $T-5$ & 15 & $T-0$ & 4 \\
$15-15$ & 38 & $15-5$ & 12 & $15-0$ & 5 \\
$10-0$ & 13 & $10-5$ & 10 & $10-0$ & 7 \\
& & $5-5$ & 12 & $5-0$ & 5 \\
\hline
\end{tabular}


c) Accroissement en haufeur des plants.

TABLEAU 7

Accroissement relatif en houteur ou cours de l'année suivant l'application du traitement Relative heigth increment during the year following transplantation

\begin{tabular}{|c|c|c|c|c|c|c|c|}
\hline \multicolumn{4}{|c|}{ Hêtre (Beech) } & \multicolumn{4}{|c|}{ Chềne (Oak) } \\
\hline $\begin{array}{l}\text { Traitement } \\
\text { (Treatment) }\end{array}$ & p. 100 & $\begin{array}{l}\text { Traitement } \\
\text { (Treatment) }\end{array}$ & p. 100 & $\begin{array}{l}\text { Traitement } \\
\text { (Treatment) }\end{array}$ & p. 100 & $\begin{array}{l}\text { Traitement } \\
\text { (Treatment) }\end{array}$ & p. 100 \\
\hline$T-t$ & 39 & $T=0$ & 17 & $T-t$ & 11 & $T-0$ & 5 \\
\hline $10-t$ & 33 & 10.0 & 15 & $10-t$ & 13 & $10-0$ & 1 \\
\hline 5-1 & 9 & $5-0$ & 0 & $5-1$ & 2 & 5.0 & 0 \\
\hline
\end{tabular}

\section{v. - Conclusions et conséquences pratiques}

\section{A. - Le rôle des racines latérales et du pivot}

Les résultats des expériences précédentes mettent en évidence le rôle non négligeable des racines latérales des plants de Chêne et de Hêtre dans la survie et le développement aérien.

Lorsqu'on sectionne les racines latérales du Hêtre au ras du pivot, celles-ci ne se régénèrent pas, ou très mal, et l'on observe une mortalité importante après la transplantation. Les racines latérales qui ont été sectionnées à quelques centimètres du pivot se régénèrent en revanche très bien ; le taux de survie est alors égal ou à peine inférieur à celui des plants à racines latérales beaucoup plus longues.

Chez les Chênes pédonculés de 3 ans, on observe le même phénomène ; mais alors que chez le Hêtre de 3 ans l'ablation des racines latérales à 5 centimètres du pivot semblait même avoir un effet bénéfique sur le développement aérien, chez le Chêne, l'ablation des racines latérales ou des pivots surnuméraires diminue le développement aérien.

Par ailleurs, en l'absence de racines latérales (traitements $T-0 ; 20-0 ; 10-0$; $5-0$ ), le développement aérien, surtout du Hêtre, est d'autant plus réduit que le sectionnement du pirot a eu lieu plus près du collet; sans doute cela est-il dû à la difficile régénération du pivot du Hêtre. Ces mutilations se traduisent aussi par des dessèchements de cimes.

II est possible aussi que le retard du débourrement soit dô à une mauvaise alimentation hydrique lorsque les racines latérales ont été supprimées. Larson et Whitmore (1970) ont en effet montré que lorsque la pression osmotique de la solution dans laquelle on élève les plants de chêne rouge augmente, ce qui dans une certaine mesure simule le déficit hydrique du sol, le débourrement est retardé. Pour une pression osmotique de -6 bars, ce retard peut atteindre une vingtaine de jours.

Jakolev (1970) expérimentant avec des semis de Chêne pédonculé, dont les racines avaient été sectionnées à différentes distances du collet avant d'être replantés en pépinière (łabl. 8) avait abouti à des conclusions analogues aux nôtres. 


$$
k A
$$




\section{TABLEAU 8}

Croissance aérienne de plants de Chêne pédonculé dont les racines ont été sectionnées a des disfances variables du collet à l'agge d'un an, d'après Jakolev (1970)

Shoot growth of oak seedlings transplanted after one year with top roots of various lengths, from Jakolev (1970)

\begin{tabular}{|c|c|c|c|c|c|}
\hline & \multicolumn{5}{|c|}{$\begin{array}{l}\text { Longueur du système racinaire d'un an } \\
\text { avant repiquage }(\mathrm{en} \mathrm{cm}) \\
\text { (Tap root length before transplanting in } \mathrm{cm} \text { ) }\end{array}$} \\
\hline & 30 & 25 & 20 & 15 & 10 \\
\hline \multicolumn{6}{|l|}{ 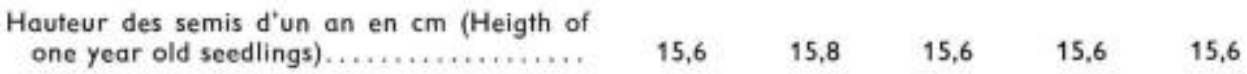 } \\
\hline \multicolumn{6}{|l|}{$\begin{array}{l}\text { Accroissement en } \mathrm{cm} \text { au cours de la (Increment } \\
\text { in } \mathrm{cm} \text { during) }\end{array}$} \\
\hline $2^{e}$ année $(2$ nd year) $\ldots \ldots \ldots \ldots \ldots \ldots$ & 6,7 & 4,7 & 3,4 & 3,3 & 2,5 \\
\hline 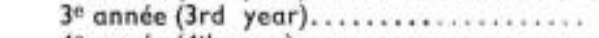 & 26,3 & 24,3 & 23,7 & 17,8 & 14,0 \\
\hline $4^{e}$ année $(4$ th year) $\ldots \ldots \ldots \ldots \ldots \ldots \ldots$ & 30,2 & 24,8 & 24,7 & 23,3 & 16,3 \\
\hline \multicolumn{6}{|l|}{ Hauteur en $\mathrm{cm}$ des plants à 4 ans (Heigth in $\mathrm{cm}$} \\
\hline at 4 years $) \ldots \ldots \ldots \ldots \ldots \ldots \ldots \ldots \ldots$ & 78,8 & 69,9 & 67,3 & 60,0 & 48,5 \\
\hline \multicolumn{6}{|l|}{ Diamètre au niveau du sol en mm à 4 ans (Stem } \\
\hline 4 years) $\ldots \ldots \ldots \ldots \ldots \ldots \ldots \ldots \ldots \ldots \ldots$ & 21 & 16,8 & 16.3 & 15,3 & 14,0 \\
\hline $\begin{array}{l}\text { Poids sec des feuilles en } g \text { d } 4 \text { ans (Leaf dry } \\
\text { weigth in } g \text { at } 4 \text { years) } \ldots \ldots \ldots \ldots \ldots \ldots \ldots\end{array}$ & 33,5 & 27,3 & 26,4 & 18,8 & 18,7 \\
\hline
\end{tabular}

II conviendrait donc de replanter les Hêtres ef les Chênes sans mutiler leurs racines ; cela n'est pas réalisable, sauf si les plants ont été élevés en conteneurs. Mais, alors on risque d'obtenir des déformations dont les conséquences fâcheuses peuvent n'apparaître qu'après plusieurs années. II faudra donc veiller :

- à ne pas arracher les racines latérales du Hêtre et de Chêne au ras du pivot, faute de quoi elles ne se régénèreraient pas ou très mal :

- à ne pas couper le pivot de Hêtre de 3 ans trop près du collet, car alors il se régénère mal :

- à concentrer au maximum le chevelu près du collet, de façon à léser le moins possible les racines au moment de l'extraction.

\section{B. - Que faire pour obtenir un chevelu plus important près du collet?}

Au début de cette note, nous avons brièvement indiqué que le milieu de culture influait essentiellement sur le développement des radicelles et non sur la « charpente» des systèmes racinaires étudiés.

En revanche, il est apparu que l'on pouvait profondément modifier le développement de la « charpente » en décapitant le pivot ou en coupant les racines latérales au ras du pivot; mais le pivot se régénère d'autant plus difficilement que la décapitation a lieu plus près du collet, ou que les plants sont plus âgés. Décapités près du collet quelques semaines après la germination, les pivots de Hêtre et de Chêne régénèrent en moyenne respectivement 5 et 3 pivots de remplacement ; aprés un an, 
ils ne régénèrent plus que 2 ou 3 racines plus ou moins obliques; après 3 ans, le pivot de Hêtre sectionné près du collet ne se régénère plus du tout, et celui du Chêne irrégulièrement. La mortalité des plants subissant ces traitements augmente aussi avec l'âge des plants.

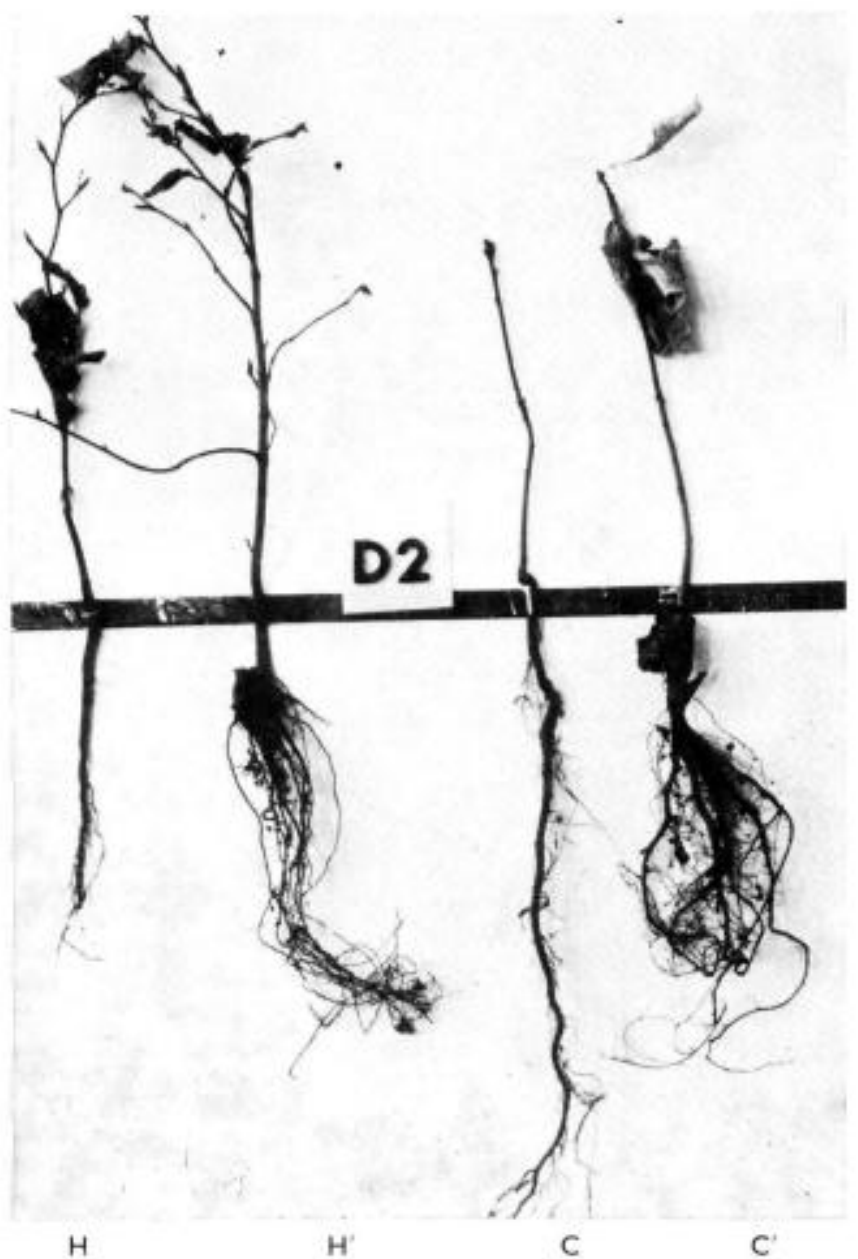

FIG. 10. - Application pratique : Plants de Hêtre $(H)$ el de Chêne $(C)$ d'un an élevés sur tourbe fertilisée. $\mathrm{H}^{\prime}$ et $\mathrm{C}^{\prime}$ ont été élevés en petit paperpof dont la base $a$ éfé coupée avant repiquage. $\mathrm{H}$ et $\mathrm{C}$ sont les témoins.

Practical application : one year old beech $(H)$ and oak seedlings grown in fertilized peat. $H^{\prime}$ and $C^{\prime}$ germinated in small poperpots which lower part was cut before transplanting.

II convient donc si l'on désire concentrer au maximum le chevelu racinaire près du collet de sectionner le pivot au cours des semaines suivant la germination (fig. 10). Deux possibilités s'offrent actuellement à nous :

1. Section de la radicule de faines ou de glands prégermés.

Les radicules de ces graines seront sélectionnées à 2-3 centimèłres du collet et à au moins 1 centimètre de la pointe du pivot. 
Dans le cas du Chêne pédonculé, dont le pivot régénère peu de racines après la décapitation, on peut être amené à resectionner les pivots de remplacement ; il faudra cependant veiller alors à éviter les déformations racinaires pouvant se produire lors du repiquage.

\section{Semis en petits paperpots.}

On fera germer les glands et les faines en petits paperpots remplis de tourbe très finement broyée ou de composts, $\mathrm{BH} 213$ pour le Hêtre (diamètre 2 centimètres4274 paperpots par mètre carré), BH 313 pour le Chêne (diamètre 3 centimètres1709 paperpots par mètre carré). Faire couper les sets de 13 centimètres de haut en deux par le revendeur). On pourrait même y conserver les graines, ne les humidifier qu'au moment de la germination. L'apport d'éléments nutritifs peut se faire soit en mélangeant l'engrais au substrat, soit par arrosage d'une solution nutrifive. La germinction pourrait ainsi être bien contrôlée et les semis gardés éventuellement jusqu'au stade deux feuilles dans ces petits paperpots dont on recoupera la base au moment du repiquage en conteneur ou sur un substrat artificiel. Pour recouper deux fois le système racinaire des semis de Chênes, on pourra couper la radicule de glands prégermés avant de les semer en paperpots.

On n'enfoncera les paperpots que de 2 centimètres dans le nouveau substrat, de façon à éviter au maximum le développement des racines latérales dans le paperpot.

L'ensemble de ces opérations (remplissage des paperpots, semis, repiquage) parait assez facilement mécanisable.

Cela est aussi simple que de repiquer des graines prégermées et ne nécessite qu'un seul tri plus commode à effectuer.

\section{C. - Conclusion}

La rupture de la radicule de faines ou de glands prégermés ne semble donc pas, ainsi que l'avait déjà souligné Mme Muller (1971) préjudiciable au développement des plants, à conditions toutefois peut-être que la cicatrisation puisse avoir lieu, ce qui implique sans doute que la température au moment du semis ne soit pas trop basse, ni l'humidité excessive.

Au stade juvénile, les systèmes racinaires à pivołs uniques du Chêne et du Hêtre peuvent être facilement transformés en systèmes racinaires à plusieurs pivots.

Cela peut être intéressant pour l'élevage de plants sur tourbe que l'on replantera avec ou sans motte; la présence de plusieurs pivots permet en effet d'améliorer l'adhésion de la tourbe aux racines.

Ces modifications présentent sans doute aussi un intérêt pour la reprise et le développement initial des plants à racines nues. Pour cela, il faudrait donc tout d'abord décapiter les pivots de semis de Chêne et de Hêtre, afin de multiplier leur nombre. Pour favoriser le développement du chevelu, il faudrait peut-être ensuite les élever sur sable. Lorsqu'ils sont élevés sur un mélange de sable $(75$ p. 100) et de tourbe $(25$ p. 100) fertilisée au début de la saison de végétation, leur croissance aérienne est, malheureusement, bien inférieure d̀ celle obtenue sur tourbe fertilisée (Le Tacon, 1974). Mais, peut-être pourra-t-on améliorer leur croissance en les arrosant tout au long de la saison de végétation avec une solution nutritive? 
Quelle sera l'influence de la morphologie juvénile des systèmes racinaires sur leur morphogénèse ultérieure, et quels autres intérêts peuvent présenter ces manipulations ?

Chez les jeunes semis, nous avons vu que lorsque l'alimentation hydrique et minérale n'étaient pas facteurs limitants, la morphogénèse n'avait pas d'influence sur la croissance aérienne.

Dans le sol, la satisfaction des besoins de la plante risque foutefois d'être limitée par la prospection du système racinaire ; il serait alors peut-être préférable que le plant dispose d'un système racinaire fasciculé permettant une bonne prospection du sol, avec quelques racines verticales permettant un bon ancrage, plutôt qu'un système racinaire d̀ un seul pivot, ou sans pivot. Peut-être n'est-il pas non plus indifférent que les racines poussent en surface ou en profondeur; les synthèses hormonales et de ce fait la croissance et la morphogénèse aérienne pourraient en effet dépendre des régimes thermiques et hydriques du sol dont on sait qu'ils varient avec la profondeur. Divers essais ont été entrepris, les uns au C.N.R.F., d'autres en collaboration avec le C.T.G.R.E.F. pour pouvoir répondre à ces questions.

Reçu pour publication en décembre 1976.

\section{Summary}

Root systems of beech and oak seedlings : 1. Modification of their morphogenesis by root tip decapitation and practical application.

To defermine the effect of root morphogenesis on various aspects of shoot development, we studied the root plasticity at various stages ; (0) just after germination, (1) at one year, and (3) et 3 years.

At stage $(0)$ the beech radicle cut once is replaced by an average of 5 roots which growth and orientation are fairly similar to that of a single taproot (fig. 2 and table 1 and 2 ).

Decapited oak radicles regenerate only an average of 3 roots very similar to that of a single tap root (fig. 3 and table 2).

When this is repeated, the average number of vertical roots increases but their diameter decreases,

At stage (1), the beech taproot cut at $10 \mathrm{~cm}$ or less from the collar is generally not replaced; a callus develops at the cut end ; oak tap roots regenerate more easily (fig. 4).

At stage (3), beech tap roots are no longer regenerated when cut at $20 \mathrm{~cm}$ or less from the collar (fig. 5 and 9) whereas oak taproots regenerate horizontal or oblique roots (fig. 7 and 9). Lateral roots, on the contrary, regenerate mostly one root with the same characteristics. At stage $(0)$ root manipulafion does not affect shoot growth (tables 1 and 2).

But later on, the root manipulations [described in \$ III. A, IV. A and in fig. 6 and 8] affect strongly mortality [table 2 for stage (1) and fig. 6 and 8 for stage (3)], date of budbreak [table 5 for stage (3)], leaf production [fable 4 for stage (1) and fig. 6 and 8 for stage (3)]. collar diameter growth [Table 6] as well as relative height growth [table 7].

Shoot development appears to be more sensifive to lateral root reduction than to tap roof shortening

The relative importance of lateral and tap roots is discussed. To concentrate the root system close to the collar it is suggested either to cut the radicle of pregerminated seeds before sowing, or to let germinate seeds in small paperpots which bottom is to be cut before transplanting (fig. 10).

Key words : Young seedlings ; Fagus sylvatica L. ; Quercus pedunculata Ehrh. ; Regeneration ; Tap root; Lateral roots ; Root/shoof relationship; Budbreak; Morfality : Shoot growth ; Nursery practices.

\section{Références bibliographiques}

CHAMPAGNAT M., BABA J., DELAUNAY M., 1974. Corrélation entre le pivot et ses ramifications dans le système racinaire de jeunes chênes cultivès sous brouillard nutritif. Rev. Cytol. el Biol. vég., 37, 407-418. 
COICY., LESAINT, C. La nutrition minérale en horticulture avancée. Revue horticole, août-sept. 1973 , no $2,316$.

DELRAN S., GARBAYE J., LE TACON F., 1975. Production rapide de plants feuillus sur tourbe fertilisée : nouveaux résultats. Revue Forestière fronçaise, n० 6, pp. 437-448.

DUSEK, 1967. The use of root pruning on Fagus sylvatica transplants. Congrès I.U.F.R.O., Münich, 1967, sect. 23 , pp. $146-159$.

JAKOLEV, 1970. Effet du cernage de plants de chêne sur leur survie ef leur croissance au cours des cinq premières années. Izv. V. U. Z. Lesn Zh, 13 (5), 162-163.

MULLER C., 1971. Influence sur la vigueur des semis, de la rupture des radicules de $\alpha$ prégermination s) de glands. Rapport interne Station d'Amélioration, C.N.R.F., sept. 1971, 5 p.

LARSON M. M., WHITMORE F. W., 1970. Moisture stress affects root regeneration and early growth of red oak seedlings. For. Sci., 16, 495-498.

RIEDACKER A., 1974. Un nouvel outil pour l'étude des racines et de la rhizosphère ; le minirhizotron. Annales des Sciences Forestières, 31 (2), 129-134.

RIEDACKER A., 1976. Etude des possibilités de modifier la morphogénèse juvénile des systèmes racinaires de quelques espèces forestières ; applications pratiques. Communication au XVIe Congrès I.U.F.R.O., Oslo, 1976, Division 1, pp. 376-387. 